\title{
Novel Index of Coronary Collateral Development as a Useful Predictor of Clinical Outcome in Type 2 Diabetic Patients With Coronary Artery Disease
}

\author{
Eue-Keun Choi, MD; Hyo-Soo Kim, MD; Kyung-Woo Park, MD; \\ Hyung-Kwan Kim, MD; Joung-Won Cho, RT; Myoung-Mook Lee, MD; \\ Young-Bae Park, MD; Yun-Shik Choi, MD
}

\begin{abstract}
Background There is a paucity of information regarding the impact of the coronary collaterals on prognosis in type 2 diabetic (T2DM) patients. We developed a novel index, which considers not only the degree of collateral circulation but also the stimulus of collateral development, and investigated its prognostic value in T2DM patients with coronary artery disease (CAD).

Methods and Results One hundred and ninety four consecutive T2DM patients were analyzed and followed for an average of 30 months. We measured the diameter stenosis (DS; \%), corrected TIMI frame count (CTFC) and Rentrop score at 3 major epicardial coronary arteries. The collateral development (CD) score was calculated by: $\Sigma$ Rentrop score +1$) \Sigma$ [DS $(\%) \times$ CTFC] $\times 1,000$. During the follow-up, acute cardiovascular events occurred in 49 patients. By multivariate analysis, the CD score was an independent predictor of adverse events not only in the total population $(\mathrm{p}<0.001$ ), but in all 3 subgroups $(\mathrm{p}=0.020$ for coronary artery bypass grafting, $\mathrm{p}=0.030$ for percutaneous transluminal coronary angioplasty (PTCA) and $\mathrm{p}=0.003$ for the medical group). Furthermore, patients in the tertile with the highest CD score showed improved survival by Kaplan-Meier analysis in the total population, the PTCA and the medical group.

Conclusions The $\mathrm{CD}$ score, a novel index of collateral development, may be a useful predictor of clinical outcome in T2DM patients with CAD. (Circ J 2005; 69: 786-792)
\end{abstract}

Key Words: Collateral circulation; Coronary artery disease; Diabetes mellitus

A number of studies have evaluated collateral circulation (CC) with intracoronary pressure measurement, which is a new technique that provides quantitative information of recruitable collateral vessels ${ }^{1-5}$ However, this method has limited applicability in patients not receiving coronary balloon angioplasty and in those with multi-vessel disease. The characteristics of coronary atherosclerosis in diabetic patients are more severe, diffuse and often occur on small vessels, which are not quite suitable to measure the intracoronary pressure gradient ${ }^{6-8}$ Also, the diabetic population is one of the few groups in which percutaneous coronary intervention does not provide survival benefit over coronary bypass graft surgery9,10

It is well known that severe coronary stenosis can increase the pressure gradient across the pre-existing collateral network resulting in the development of CC!1-13 Without directly measuring the intracoronary pressure gradient, we assumed the severity of coronary artery stenosis to be a surrogate marker that represents the stimulation of collateral development. We also presumed that the impaired responsiveness to the transcollateral pressure gradient might

(Received July 28, 2004; revised manuscript received April 1, 2005; accepted April 7, 2005)

Department of Internal Medicine, Seoul National University College of Medicine, Cardiovascular Laboratory Clinical Research Institute, Seoul National University Hospital, Seoul, Korea

Mailing address: Hyo-Soo Kim, MD, Department of Internal Medicine, Seoul National University College of Medicine, 28 YongonDong, Chongno-Gu, Seoul 110-744, Korea. E-mail: hyosoo@snu. ac.kr have clinical significance. There are many published studies which have reported diabetic patients to have impaired CC development, but it is unknown what effects this has on outcomes! ${ }^{14,15}$ Therefore, we investigated the impact of the degree of CC development on the prognosis of type 2 diabetic (T2DM) patients with coronary artery disease $(\mathrm{CAD})$ using a novel index which represents the responsiveness of the collateral vasculature to collateral stimulation.

\section{Methods}

\section{Patient Population}

We enrolled 642 consecutive diabetes mellitus (DM) patients who underwent coronary angiography at the Seoul National University Hospital between June 1998 and April 2001. Patients greater than 40 years of age with epicardial coronary artery stenosis $\geq 75 \%$ with either symptomatic angina for more than 1 month or reversible myocardial ischemia on a non-invasive stress test were selected. Those with type 1 diabetes, myocardial infarction within the previous 3 months, or previous coronary intervention (percutaneous transluminal coronary angioplasty (PTCA) or coronary artery bypass grafting (CABG)) were excluded. A total of 210 patients met the criteria and were followed until 30 June 2002. Sixteen patients were lost during follow-up leaving 194 patients for analysis. As revascularization is probably the most important prognostic factor when dealing with patients having $\mathrm{CAD}$, the enrolled patients were subgrouped and further analyzed by the therapy 
Table 1 Baseline Clinical and Angiographic Characteristics According to Revascularization Therapy of Diabetic Patients

\begin{tabular}{|c|c|c|c|c|}
\hline Characteristic & $\begin{array}{c}C A B G \\
(n=62)\end{array}$ & $\begin{array}{c}P T C A \\
(n=69)\end{array}$ & $\begin{array}{c}\text { Medical } \\
(n=63)\end{array}$ & p-value \\
\hline Age (years) & $62.0 \pm 7.7$ & $62.2 \pm 7.2$ & $63.1 \pm 7.5$ & 0.67 \\
\hline Male & $62.9 \%(39)$ & $58.0 \%(40)$ & $60.3 \%(38)$ & 0.85 \\
\hline$B M I\left(\mathrm{~kg} / \mathrm{m}^{2}\right)$ & $25.3 \pm 2.8$ & $24.8 \pm 3.0$ & $24.6 \pm 3.2$ & 0.43 \\
\hline Duration of angina (months) & $13.0 \pm 18.8$ & $8.6 \pm 13.8$ & $22.9 \pm 23.7$ & 0.002 \\
\hline \multicolumn{5}{|l|}{ Clinical manifestation } \\
\hline Stable angina pectoris & $27.4 \%(17)$ & $33.3 \%(23)$ & $25.4 \%(16)$ & 0.58 \\
\hline Unstable angina pectoris & $53.2 \%(33)$ & $53.6 \%(37)$ & $28.6 \%(18)$ & 0.005 \\
\hline Silent myocardial ischemia & $19.4 \%(12)$ & $13.0 \%(9)$ & $46.0 \%(29)$ & $<0.001$ \\
\hline Hypertension & $75.8 \%(47)$ & $58.0 \%(40)$ & $81.0 \%(51)$ & 0.009 \\
\hline Smoking & $33.9 \%(21)$ & $30.4 \%(21)$ & $41.3 \%(26)$ & 0.42 \\
\hline Hypercholesterolemia & $58.1 \%(36)$ & $44.9 \%(31)$ & $46.0 \%(29)$ & 0.26 \\
\hline LV dysfunction & $14.5 \%(9)$ & $2.9 \%(2)$ & $12.7 \%(8)$ & 0.05 \\
\hline Fasting blood glucose $(\mathrm{mg} / \mathrm{dl})$ & $146.7 \pm 44.2$ & $164.2 \pm 46.9$ & $163.4 \pm 60.8$ & 0.10 \\
\hline $\mathrm{HbAlc}_{\mathrm{s}}(\%)$ & $7.9 \pm 1.3$ & $8.2 \pm 1.5$ & $8.1 \pm 1.3$ & 0.57 \\
\hline Duration of DM (years) & $11.7 \pm 7.3$ & $9.5 \pm 8.1$ & $13.5 \pm 7.5$ & 0.01 \\
\hline \multicolumn{5}{|l|}{ Medication } \\
\hline Insulin & $30.6 \%(19)$ & $24.6 \%(17)$ & $57.1 \%(36)$ & $<0.001$ \\
\hline Oral hypoglycemic agent & $62.9 \%(39)$ & $62.3 \%(43)$ & $38.1 \%(24)$ & 0.006 \\
\hline Diet only & $6.5 \%(4)$ & $13.0 \%(9)$ & $4.8 \%(3)$ & 0.19 \\
\hline Aspirin & $100 \%(62)$ & $94.2 \%(65)$ & $92.1 \%(58)$ & 0.09 \\
\hline$\beta$-blocker & $40.3 \%(25)$ & $37.7 \%(26)$ & $36.5 \%(23)$ & 0.90 \\
\hline Calcium channel blocker & $85.5 \%(53)$ & $75.4 \%(52)$ & $71.4 \%(45)$ & 0.15 \\
\hline Nitrates & $90.3 \%(56)$ & $81.2 \%(56)$ & $76.2 \%(48)$ & 0.11 \\
\hline Lipid lowering agent & $56.5 \%(35)$ & $44.9 \%(31)$ & $46.0 \%(29)$ & 0.14 \\
\hline$A C E I$ or $A R B$ & $27.4 \%(17)$ & $24.6 \%(17)$ & $41.3 \%(26)$ & 0.09 \\
\hline Proximal lesion location & $66.1 \%(41)$ & $44.9 \%(31)$ & $63.5 \%(40)$ & 0.03 \\
\hline No. of total occlusion & $46.8 \%(29)$ & $20.3 \%(14)$ & $31.7 \%(20)$ & 0.01 \\
\hline \multicolumn{5}{|l|}{ No. of diseased vessel } \\
\hline 1 & $3.2 \%(2)$ & $36.2 \%(25)$ & $7.9 \%(5)$ & $<0.001$ \\
\hline 2 & $21.0 \%$ & $37.7 \%(26)$ & $25.4 \%(16)$ & 0.13 \\
\hline 3 & $75.8 \%(47)$ & $26.1 \%(18)$ & $66.7 \%(42)$ & $<0.001$ \\
\hline
\end{tabular}

BMI, body-mass index; LV dysfunction, left ventricular dysfunction; HbAlc, glycosylated hemoglobin; DM, diabetes mellitus; ACEI, angiotensin converting enzyme inhibitor; $A R B$, angiotensin receptor blocker.

they received at the time of angiography (62 for $\mathrm{CABG}$ group, 69 for PTCA group and 63 for the medical group).

\section{Clinical Data}

Gender, age, smoking status, body-mass index, duration of diabetes, current treatment for diabetes, the presence of hypertension, and family history of ischemic heart disease were recorded. Fasting plasma glucose level, glycosylated hemoglobin level, and fasting lipid profiles were obtained.

Diabetes was defined as a fasting plasma glucose level $\geq 126 \mathrm{mg} / \mathrm{dl}$ or if the subjects were treated with dietary modification, oral hypoglycemic agents, or insulin. The duration of diabetes was estimated from the detailed review of the patients' clinical charts. Hypertension was defined as 2 measurements of the systolic/diastolic blood pressure over $140 / 90 \mathrm{mmHg}$, or treatment with antihypertensive medications. Hypercholesterolemia was defined as fasting serum total cholesterol $\geq 240 \mathrm{mg} / \mathrm{dl}$ or if the subjects were being treated with lipid-lowering agents or dietary modification. Left ventricular dysfunction was defined as resting left ventricular ejection fraction $<40 \%$ by echocardiography.

\section{Angiographic Analysis and the Development of a Novel Index of Coronary Circulation}

We used 2 different methods to measure the luminal diameter stenosis. The eye-balling technique was applied to measure the total coronary atherosclerosis burden, and a quantitative coronary angiography (QCA) analysis was used to measure the critical lesion that influences coronary
CC development. First, we graded the diameter stenosis of the epicardial coronary arteries and their secondary branches were visually divided into 15 segments 16 Each segment was graded as 1 of 4 levels $(50,75,90$, and $100 \%)$ and stenosis smaller than $50 \%$ were graded as irregularities which were taken as $40 \%$ stenosis, 6,17 When diffuse stenoses were present on the same segment of the coronary artery, the grade of the narrowest one was considered. The atherosclerosis (ATS) score was calculated by summing the percentage narrowing of all segments, including multiple stenosis along the same main vessel and those of its secondary branches 6 To validate the visual grading system, we selected 94 stenosis segments at random and compared data from visual grading system and the reading obtained by the QCA. The values coded as irregularities, 50, 75 and $90 \%$ by the visual grading system corresponded to $44.3 \pm$ 4.6, 49.7 $\pm 5.9,71.2 \pm 8.0,84.2 \pm 9.6$ narrowing, respectively, on quantitative angiography. Significant obstruction of the coronary vessel was defined as stenosis more than $50 \%$ compared to the prestenotic segment and these definitions constituted the basis for the diagnosis of 1-vessel, 2-vessel, or 3-vessel disease.

Coronary angiograms were analyzed using online QCA system (Quantcor QCA Version 4.0, Pie Medical Imaging, Maastricht, The Netherlands). Angiographic measurements were made during diastole after the administration of intracoronary nitroglycerin, using the guiding catheter as a reference. We measured the percentage diameter stenosis and the minimal luminal diameter of the atherosclerotic lesion and selected lesions which had more than 75\% diam- 
Table 2 Angiographic Values According to Revascularization Therapy of Diabetic Patients

\begin{tabular}{lcccc}
\hline \hline Characteristic & $\begin{array}{c}\text { CABG } \\
(n=62)\end{array}$ & $\begin{array}{c}\text { PTCA } \\
(n=69)\end{array}$ & $\begin{array}{c}\text { Medical } \\
(n=63)\end{array}$ & $p$-value \\
\hline ATS score & $397.7 \pm 124.5$ & $263.0 \pm 118.6$ & $390.2 \pm 145.1$ & $<0.001$ \\
LAD & $151.0 \pm 59.3$ & $101.9 \pm 67.7$ & $139.0 \pm 69.5$ & $<0.001$ \\
LCX & $124.6 \pm 69.1$ & $68.6 \pm 61.8$ & $128.8 \pm 74.2$ & $<0.001$ \\
MLDS $(122)$ & $122.0 \pm 64.1$ & $92.5 \pm 68.5$ & $122.5 \pm 72.1$ & 0.02 \\
LAD & $202.8 \pm 64.8$ & $135.5 \pm 63.8$ & $177.2 \pm 73.3$ & $<0.001$ \\
LCX & $90.4 \pm 8.7$ & $87.1 \pm 8.3$ & $89.6 \pm 8.7$ & 0.17 \\
$R C A$ & $87.1 \pm 9.5$ & $89.5 \pm 9.1$ & $89.2 \pm 9.5$ & 0.48 \\
CTFC, frames & $90.0 \pm 9.5$ & $89.0 \pm 8.0$ & $89.2 \pm 9.7$ & 0.79 \\
LAD & & & & \\
LCX & $41.2 \pm 36.5$ & $25.8 \pm 22.9$ & $34.7 \pm 33.8$ & 0.07 \\
$R C A$ & $36.1 \pm 31.7$ & $44.2 \pm 36.3$ & $37.6 \pm 32.9$ & 0.59 \\
CS score & $41.8 \pm 36.0$ & $34.3 \pm 27.5$ & $35.2 \pm 33.5$ & 0.51 \\
LAD & $86.0 \pm 57.8$ & $48.1 \pm 48.4$ & $67.9 \pm 56.8$ & $<0.001$ \\
LCX & $33.1 \pm 37.2$ & $14.2 \pm 21.3$ & $21.5 \pm 32.1$ & 0.002 \\
$R C A$ & $24.4 \pm 31.7$ & $16.0 \pm 31.2$ & $24.0 \pm 32.6$ & 0.23 \\
Rentrop score & $28.5 \pm 36.1$ & $17.9 \pm 26.4$ & $22.3 \pm 32.2$ & 0.16 \\
LAD & $1.76 \pm 1.76$ & $0.70 \pm 1.34$ & $1.06 \pm 1.98$ & 0.002 \\
LCX & $0.68 \pm 1.20$ & $0.26 \pm 0.72$ & $0.33 \pm 1.27$ & 0.07 \\
$R C A$ & $0.27 \pm 0.79$ & $0.17 \pm 0.66$ & $0.16 \pm 0.57$ & 0.59 \\
CD score & $0.81 \pm 1.40$ & $0.16 \pm 0.56$ & $0.57 \pm 1.47$ & 0.009 \\
\hline
\end{tabular}

$L A D$, left anterior descending coronary artery; LCX, left circumflex coronary artery; RCA, right coronary artery; ATS score, atherosclerosis score; MLDS, maximal luminal diameter stenosis CTFC, corrected TIMI frame count, CS (Collateral Stimulation) score $=\Sigma$ (Maximal luminal diameter stenosis $\times C T F C), C D$ (Collateral Development) score $=(\Sigma$ Rentrop score +1$) / C S$ score $\times$ 1,000 .

Table 3 Acute Cardiovascular Events During Follow-up

\begin{tabular}{lccc}
\hline \hline & $C A B G(n=62)$ & $P T C A(n=69)$ & Medical $(n=63)$ \\
\hline Cardiovascular death (\%) & $3(4.8)$ & $1(1.4)$ & $11(17.5)$ \\
Nonfatal myocardial infarction (\%) & $1(1.6)$ & $1(1.4)$ & $2(3.2)$ \\
Unstable angina (\%) & $7(11.3)$ & $17(24.6)$ & $6(9.5)$ \\
& $11(17.7)$ & $19(27.5)$ & $19(30.2)$ \\
\hline
\end{tabular}

$C A B G$, coronary artery bypass grafting; PTCA, percutaneous transluminal coronary angioplasty.

eter stenosis. When multiple stenoses were present on the same epicardial coronary artery and its secondary branches, the percentage diameter stenosis was measured from the narrowest portion. Finally, 372 lesions were selected and analyzed.

The antegrade flow of each epicardial coronary arteries with more than $75 \%$ diameter stenosis was measured by the Thrombolysis In Myocardial Infarction (TIMI) frame count. The CTFC (corrected TIMI frame count) was the number of cine frames required for contrast to first reach standardized distal coronary landmarks in the culprit artery and was measured on a cine viewer. Cine film speed was 30 frames/s. A frame count of 100 , a value that is the $99^{\text {th }}$ percentile of patent vessel, was imputed to an occluded vessel ${ }^{18}$

Collateral flow before revascularization was graded using the classification developed by Rentrop et al: grade $0=$ no visible filling of any collateral channels; $1=$ filling of side branches of the infarct artery with no dye reaching the epicardial segment; 2 =partial filling of the epicardial vessel; and $3=$ complete filling of the epicardial vessel by collateral vessels 19

With these data, we went on to develop a novel index of $\mathrm{CC}$ taking into account not only the degree of collateral vessels, but also the stimulus of collateral development. The degree of collateral stimulation was quantified using a collateral stimulation (CS) score, which was calculated by summing the product of diameter stenosis $(\%)$ and CTFC of each 3 major coronary arteries. The novel index was termed 'collateral development (CD) score', which was calculated by dividing the sum of the Rentrop score by the CS score and multiplied by 1,000.

$$
\begin{aligned}
& \text { CD score }=(\Sigma \text { Rentrop score }+1) / \Sigma \text { diameter stenosis }(\%) \\
& \times \text { CTFC }] \times 1,000
\end{aligned}
$$

\section{Data Collection and Long-Term Follow-up Outcome}

Baseline demographic, clinical, angiographic, and procedural data including complications were recorded on standardized forms by physicians and entered into a computerized database. The following clinical events were considered as acute cardiovascular events (ACE): cardiovascular death, non-fatal myocardial infarction, and unstable angina resulting in hospitalization. Cardiovascular death was defined as sudden death, acute left ventricular failure, fatal myocardial infarction, and death during or immediately after coronary bypass surgery or cerebral infarction. If a patient experienced more than 1 event during follow-up, only the first was considered in the analysis.

Long-term follow-up data was obtained from medical records and by telephone contact with trained personnel. Patients were asked to provide details of symptoms and the reasons for all hospitalizations, the occurrence of myocardial infarction, and additional angioplasty or coronary 
Table 4 Clinical Variables Related to CD Score

\begin{tabular}{lcc}
\hline \hline Variable & CD score & p value \\
\hline Duration of diabetes & $R=-0.101$ & 0.162 \\
Fasting blood glucose & $R=-0.059$ & 0.412 \\
Glycosylated hemoglobin, HbAlc & $R=-0.161$ & 0.031 \\
Treatment modality of DM & & \\
$\quad$ Non-insulin $(n=122)$ & $50.7 \pm 32.9$ & 0.017 \\
$\quad$ Insulin $(n=72)$ & $39.5 \pm 28.2$ & \\
\end{tabular}

$C D$, collateral development; DM, diabetes mellitus.

surgery. Clinical follow-up (mean, 29.9 \pm 12.4 months) was available in $92 \%$ of cases.

\section{Statistical Analysis}

Continuous variables are expressed as mean $\pm \mathrm{SD}$, whereas categorical variables are presented as absolute values and percentages. Group differences of continuous variables were tested by analysis of variance and followed by a Scheffe's post-hoc test. Group differences of categorical variables were analyzed by a chi-square or Fisher's exact test. The Cox proportional hazard stepwise regression analysis was used to determine the univariate and multivariate relationships between clinical variables and clinical outcomes. The variables used for the analysis included age, gender, obesity, smoking, hypertension, hypercholesterolemia, left ventricular dysfunction, regional wall motion abnormality of the left ventricle, multi-vessel disease, proximal lesion, chronic total occlusion, CC (Rentrop score), CD score, and the ATS score. Survival curves were generated using the Kaplan-Meier method. Comparisons between survival curves were performed using the log-rank test. A value of $p<0.05$ was considered statistically significant and the SPSS statistical software package version 10.0 (Chicago, IL, USA)was used to perform all statistical calculations.

\section{Results}

\section{Baseline Characteristics}

The clinical characteristics of the patients are listed in Table 1 . Of these patients, only $8 \%$ were treated with diet alone, $37 \%$ with insulin, and $55 \%$ with oral hypoglycemic agents. The mean duration of diabetes and glycated hemoglobin level were $13.5 \pm 7.5$ years and $8.1 \pm 1.3 \%$, respectively. Fifty-six patients $(29 \%)$ had angiographic evidence of CC flow grade $>1$.

Patients in the medical group differed from those who received revascularization in several ways. Patients in the medical group had significantly longer duration of angina and diabetes and a greater incidence of silent myocardial ischemia, insulin treatment, as well as a lower incidence of unstable angina (Table 1). For angiographic parameters, patients in the PTCA group had a lower incidence of proximal lesion location and a lower ATS score, CS score and Rentrop score. Fewer patients in the PTCA group had total occlusion or multi-vessel disease compared to the other 2 groups (Tables 1,2). However, the CD score between the 3 groups showed no significant difference.

\section{Clinical Outcomes}

During the mean duration of follow-up of 30 months, 49 patients experienced ACE (annual patients event rate= $49 /[194 \times 2.5]=10.1 \%$ ) (Table 3 ). Fifteen of these patients

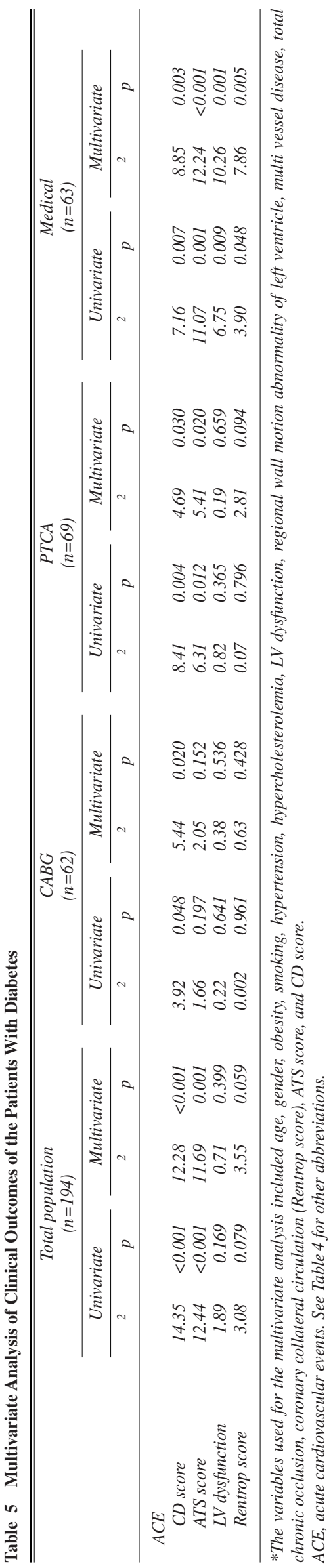


A

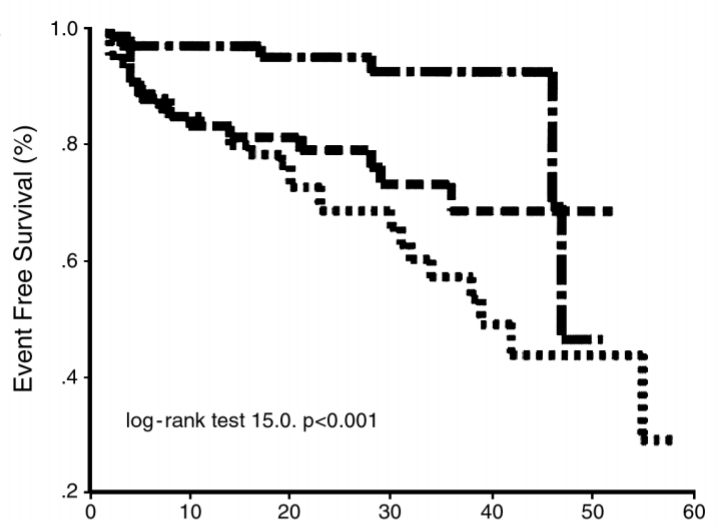

$\mathbf{C}$

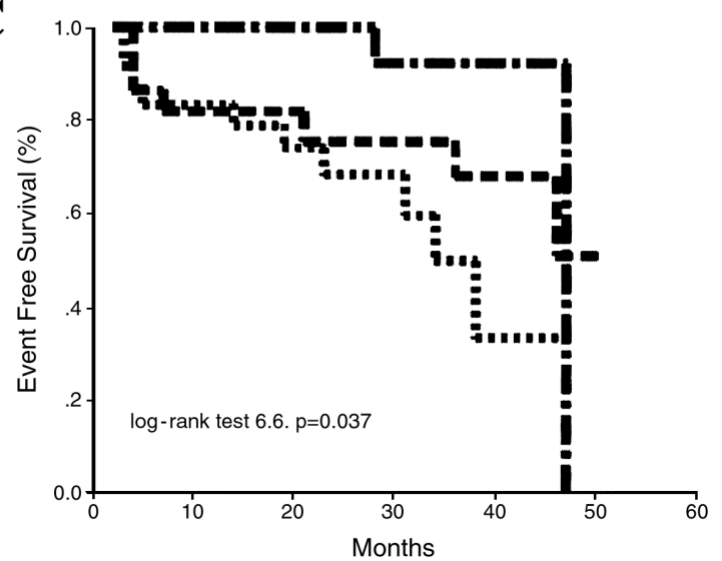

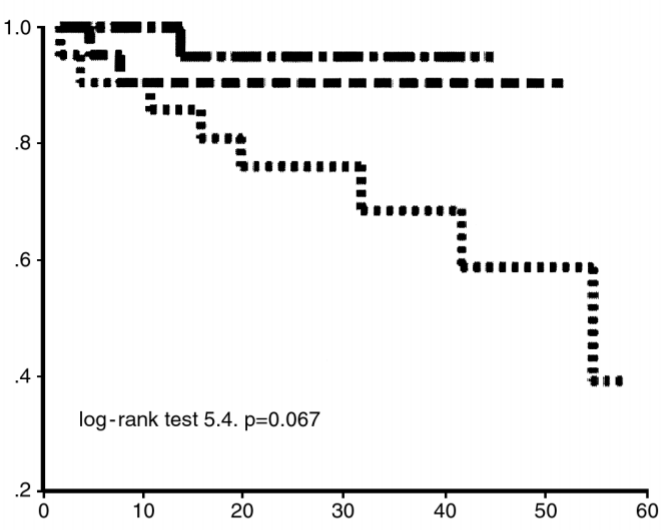

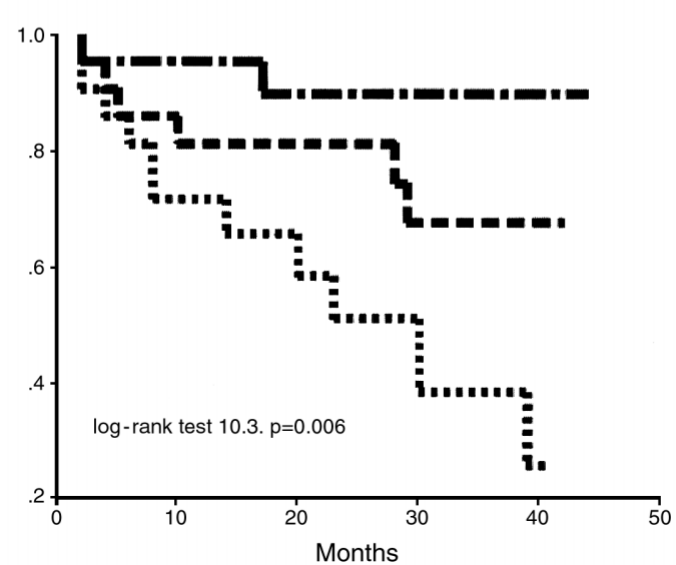

$\mathbf{B}$

D

Fig 1. Relationship between degree of coronary collateral development and acute cardiovascular events. Kaplan-Meier analysis demonstrating proportion of patients surviving from acute cardiovascular events during follow-up period. Study cohort is divided into tertiles according to CD score in the total population (A). CABG group (B), PTCA group (C), and the medical group (D). - ____, represents tertile with highest CD score; ____- , tertile with intermediate CD score; ------., tertile with lowest CD score.

had 2 or more acute events. Noncardiovascular causes of death included malignancy (1) and postoperative complication (1).

Patients in the CABG group were less likely to experience ACE compared with patients in the medical group (log-rank test $4.3, p=0.04$ ), while the data was non-significant when compared with those in the PTCA group $(\mathrm{p}=0.09)$.

\section{Clinical Variables Related to CD Score}

Of the various clinical factors of diabetes studied, glycosylated hemoglobin level and insulin treatment were significantly associated with the CD score, while the duration of diabetes and the fasting glucose level were not (Table 4).

\section{Degree of Collateral Development and Acute Cardiovascular Event}

We analyzed the degree of collateral development and ACE in the total population, and performed subgroup analyses according to revascularization therapy.

The CD and ATS scores were found to be univariate predictors of ACE by Cox proportional hazard analysis. However, after multivariate analysis adjusted with other covariates, only the $\mathrm{CD}$ score remained as a significant independent predictor of acute events in the total population and in all 3 subgroups, whereas the ATS score was a significant predictor in the PTCA and medical groups, but not in the CABG group (Table 5). The left ventricular dysfunction and Rentrop score were also significantly related to acute events in the medical group.

The CD score was significantly lower in patients with acute events during follow-up compared with those without events (CD score 30.40 vs $51.99,23.81$ vs 46.30, 36.53 vs $58.49,28.08$ vs 51.19 in patients with acute events vs without events in total population, the CABG, PTCA and medical group, respectively, all $\mathrm{p}<0.05)$. To study the relationship between the degree of $\mathrm{CD}$ and ACE, the study population was divided into tertiles according to the $\mathrm{CD}$ score. Using the Kaplan-Meier analysis, event-free survival from ACE was significantly associated with the CD score in the entire population, the PTCA group, and the medical group $(\mathrm{p}<0.001$ for total population, $\mathrm{p}=0.067$ for $\mathrm{CABG}$ group, $\mathrm{p}=0.037$ for PTCA group and $\mathrm{p}=0.006$ for the medical group). As expected, the outcome was worse in those with a less developed CC (Fig 1). In contrast, when the population was similarly divided into tertiles according to ATS score, there was no association between event-free survival from ACE and ATS score in all 3 subgroups $(p=0.26$ for $C A B G$ group, $p=0.13$ for PTCA group and $\mathrm{p}=0.15$ for the Medical group by Kaplan-Meier analysis). In addition, when the population was divided by simple presence or absence of CC, we found no significant association between event-free survival from ACE and evidence of CC. 


\section{Discussion}

The major findings of the present study among T2DM patients with CAD show: (1) the CD score, a novel index of CC development taking into account not only the growth of new collaterals, but also the stimulus they are responding to is significantly related to ACE, including cardiovascular death, myocardial infarction, and unstable angina after adjustment for other cardiac risk factors; and (2) the CD score is inversely related with the severity of DM (control of diabetes and insulin treatment). The CD score was a significant predictor of ACE not only in patients followed medically, but also in patients that received PTCA or CABG suggesting that this novel index is of prognostic significance irrespective of revascularization therapy. To the best of our knowledge, this is the first published study on the correlation between CC development and clinical outcomes in T2DM patients and could provide insight into the importance of not only the development of new vessels, but also the stimuli that the new vessels are responding to when considering the clinical significance of collateral formation in these patients.

\section{Introduction of a New Index, the CD Score}

In the process of developing this new index, we took various factors into account. The severity of coronary artery stenosis and the duration of myocardial ischemic symptoms are well-established factors for determining the extent of collateralization.19-21 However, we could not adjust for the duration of ischemia since diabetic patients often have silent myocardial ischemia, making it almost impossible to determine the exact onset of ischemia. Instead, to account for the extent of collateralization, we added antegrade flow of the recipient coronary arteries as a factor, because in patients with TIMI grade 3 perfusion, the absence of angiographically visible CC does not reliably reflect the anatomic absence of collateral vessels ${ }^{22}$ Moreover, only patients expected to have experienced sufficient ischemic time to stimulate collateral vessels were included, whereas those who had insufficient time to mature welldeveloped collateral vessels (eg, those with an acute myocardial infarction) were excluded. Because collateral artery proliferation is not a process of passive dilatation, but rather one of active proliferation and remodeling, these vessels need time to grow and mature? 3

\section{Score, a Very Important Predictor of Cardiovascular Events in Diabetic Patients}

Reports on the correlation between coronary collaterals and prognosis are controversial.24-26 It has been reported that the presence of coronary collateral vessels at the onset of myocardial infarction is associated with a reduced infarct size, with improved ventricular function, and decreased cardiogenic shock and in-hospital death24,25 However, a recent study has reported that the pre-intervention angiographic evidence of coronary collateral vessels after acute myocardial infarction had no significant protective effect ${ }^{26}$ In our study, which excluded patients with recent myocardial infarction, the simple presence of CC had no significant relationship with a future cardiac event. The reason for this might be that $\mathrm{CC}$ is dependent on stenosis and antegrade flow in recipient vessels. Therefore, without considering these factors, the simple presence of CC might have a weak prognostic value. Our findings conclusively reveal that the ratio of the collateral stimulation to collateral devel- opment, ie, the CD score, is a significant prognostic factor in T2DM patients with CAD.

Natali et $\mathrm{al}^{6}$ reported that the ATS score is an independent predictor of cardiac death in T2DM patients. In our study, the ATS score was a univariate and multivariate predictor of ACE, with the exception of the CABG group. However, event free survival from ACE was associated only with the CD score using the Kaplan-Meier analysis. Our results show that the response to collateral stimulation (ie, CD score) has more prognostic value than simple ischemic stress (ie, ATS score) especially in diabetic patients who are suspected of having decreased collateral development response.

\section{Clinical Parameters of Diabetes Associated With the CD Score}

No previously published reports have shown that the severity of DM is significantly related to coronary collaterals in T2DM patients with CAD. A recent study on animals demonstrated that chronic hyperglycemia exerts a negative effect on the development of coronary $\mathrm{CC}^{27}$ Our study is the first to demonstrate that diabetic patients with poor glucose control have a lower CD score and a poor cardiac outcome.

\section{Study Limitations}

One limitation of the present study is that patients with acute myocardial infarction were excluded from the study. Therefore, our results cannot be generalized in diabetic patients with acute total occlusion of coronary. Another limitation is the small study population and relative short duration of follow-up. At last, data on the cause of events were obtained retrospectively. In conclusion, the CD score, a novel index of CD, is a useful predictor of ACE in T2DM patients with CAD, and is clinically significant irrespective of revascularization therapy. Therefore, assessment of the CD score in patients with T2DM patients may help identify subgroups of patients at high risk for future adverse cardiac events.

\section{Acknowledgment}

This study was supported by a grant for the Korea Health 21 R\&D Project, Ministry of Health \& Welfare, Republic of Korea (0412-CR02-07040001).

\section{References}

1. Sakai K, Yamagata T, Teragawa H, Matusuura H, Chayama K. Nicorandil enhances myocardial tolerance to ischemia without progressive collateral recruitment during coronary angioplasty. Circ J 2002; 66: 317-322

2. Pijls NH, Bech GJ, el Gamal MI, Bonnier HJ, De Bruyne B, Van Gelder B, et al. Quantification of recruitable coronary collateral blood flow in conscious humans and its potential to predict future ischemic events. J Am Coll Cardiol 1995; 25: 1522-1528.

3. Watarai M, Takatsu F, Horibe H, Yanase M, Takemoto K, Shimizu $\mathrm{S}$, et al. Myocardial ischemia during percutaneous transluminal coronary angioplasty in patients with rich collateral circulation of the target lesion. Circ J 2002; 66: 534-536.

4. van Liebergen RA, Piek JJ, Koch KT, de Winter RJ, Schotborgh CE, Lie KI. Quantification of collateral flow in humans: A comparison of angiographic, electrocardiographic and hemodynamic variables. $J$ Am Coll Cardiol 1999; 33: 670-677.

5. Seiler C, Fleisch M, Garachemani A, Meier B. Coronary collateral quantitation in patients with coronary artery disease using intravascular flow velocity or pressure measurements. J Am Coll Cardiol 1998; 32: $1272-1279$.

6. Natali A, Vichi S, Landi P, Severi S, L'Abbate A, Ferrannini E. Coronary atherosclerosis in Type 2 diabetes: Angiographic findings and clinical outcome. Diabetologia 2000; 43: 632-641. 
7. Ammann P, Brunner-La Rocca H, Fehr T, Munzer T, Sagmeister M, Angehrn W, et al. Coronary anatomy and left ventricular ejection fraction in patients with type 2 diabetes admitted for elective coronary angiography. Catheter Cardiovasc Interv 2004; 62: 432-438.

8. Elezi S, Kastrati A, Pache J, Wehinger A, Hadamitzky M, Dirschinger $\mathrm{J}$, et al. Diabetes mellitus and the clinical and angiographic outcome after coronary stent placement. J Am Coll Cardiol 1998; 32: $1866-$ 1873.

9. The Bypass Angioplasty Revascularization Investigation (BARI) Investigators. Comparison of coronary bypass surgery with angioplasty in patients with multivessel disease. $N$ Engl J Med 1996; 335: $217-$ 225.

10. King SB III, Kosinski AS, Guyton RA, Lembo NJ, Weintraub WS. Eight-year mortality in the Emory Angioplasty versus Surgery Trial (EAST). J Am Coll Cardiol 2000; 35: 1116-1121.

11. Pohl T, Seiler C, Billinger M, Herren E, Wustmann K, Mehta H, et al. Frequency distribution of collateral flow and factors influencing collateral channel development: Functional collateral channel measurement in 450 patients with coronary artery disease. J Am Coll Cardiol 2001; 38: 1872-1878.

12. Cohen M, Sherman W, Rentrop KP, Gorlin R. Determinants of collateral filling observed during sudden controlled coronary artery occlusion in human subjects. J Am Coll Cardiol 1989; 13: 297-303.

13. Fujita M, Tambara K. Recent insights into human coronary collateral development. Heart 2004; 90: 246-250.

14. Abaci A, Oguzhan A, Kahraman S, Eryol NK, Unal S, Arinc H, et al. Effect of diabetes mellitus on formation of coronary collateral vessels. Circulation 1999; 99: 2239-2242.

15. Waltenberger J, Lange J, Kranze A. Vascular endothelial growth factor-A-induced chenotaxis of monocytes is attenuated in patients with diabetes mellitus: A potential predictor for the individual capacity to develop collaterals. Circulation 2000; 102: 185-190.

16. Austen WG, Edwards JE, Frye RL, Gensini GG, Gott VL, Griffith $\mathrm{LS}$, et al. A reporting system on patients evaluated for coronary artery disease: Report of the Ad Hoc Committee for Grading of Coronary Artery Disease, Council on Cardiovascular Surgery, American Heart Association. Circulation 1975; 51: 5-40.

17. Rowe GG, Thomsen JH, Stenlund RR, McKenna DH, Sialer S, Corliss RJ. A study of hemodynamics and coronary blood flow in man with coronary artery disease. Circulation 1969; 39: 139-148.
18. Gibson CM, Cannon CP, Daley WL, Dodge JT Jr, Alexander B Jr, Marble SJ, et al. TIMI frame count: A quantitative method of assessing coronary artery flow. Circulation 1996; 93: 879-888.

19. Rentrop KP, Cohen M, Blanke H, Phillips RA. Changes in collateral channel filling immediately after controlled coronary artery occlusion by an angioplasty balloon in human subjects. J Am Coll Cardiol 1985; 5: 587-592.

20. Piek JJ, Koolen JJ, Hoedemaker G, David GK, Visser CA, Dunning AJ. Severity of single-vessel coronary arterial stenosis and duration of angina as determinants of recruitable collateral vessels during balloon angioplasty occlusion. Am J Cardiol 1991; 67: 13-17.

21. Matsuo H, Watanabe S, Segawa T, Hitose T, Iwama M, Tanaka S, et al. Evidence of a cellular protective effect by antecedent angina independent of collateral flow recruitment during coronary angioplasty in humans. Circ J 2002; 66: $741-745$.

22. Yoshida N, Fujita M, Yamanishi K, Miwa K. Relation between collateral channel filling and flow grade in recipient coronary arteries in patients with stable effort angina. J Am Coll Cardiol 1993; 22: 426-430.

23. Schaper W, Buschmann I. Collateral circulation and diabetes. Circulation 1999; 99: 2224-2226.

24. Habib GB, Heibig J, Forman SA, Brown BG, Roberts R, Terrin ML, et al. Influence of coronary collateral vessels on myocardial infarct size in humans, results of phase I thrombolysis in myocardial infarction (TIMI) trial. Circulation 1991; 83: 739-746.

25. Perez-Castellano N, Garcia EJ, Abeytua M, Soriano J, Serrano JA, Elizaga J, et al. Influence of collateral circulation on in-hospital death from anterior acute myocardial infarction. J Am Coll Cardiol 1998; 31: $512-518$

26. Antoniucci D, Valenti R, Moschi G, Migliorini A, Trapani M, Santoro GM, et al. Relation between preintervention angiographic evidence of coronary collateral circulation and clinical and angiographic outcomes after primary angioplasty or stenting for acute myocardial infarction. Am J Cardiol 2002; 89: $121-125$.

27. Weihrauch D, Lohr NL, Mraovic B, Ludwig LM, Chilian WM, Pagel PS, et al. Chronic hyperglycemia attenuates coronary collateral development and impairs proliferative properties of myocardial interstitial fluid by production of angiostatin. Circulation 2004; 109: 2343-2348. 